\title{
Panum's fusional area estimated with a criterion-free technique
}

\author{
THOMAS HECKMANN and CLIFTON M. SCHOR \\ University of California, Berkeley, California
}

\begin{abstract}
It has been reported that criterion-free estimates of the upper disparity limits for fusion of line targets are small enough to be accounted for by monocular vernier sensitivity. However, targets such as lines, which contain high spatial frequencies, may ensure small fusion limits, since fusion limits obtained with criterion-dependent methods for narrow-band targets, such as sinusoids or difference-of-Gaussian luminance profiles, are proportional to target spatial periods. Experiment 1 therefore explored whether criterion-free methods give fusion limits for narrow-band targets that can be accounted for by vernier sensitivity. Vertical fusion limits were estimated by a method that forced observers to discriminate a disparate sinusoidal grating from an immediately adjacent zero-disparity grating. Fusion limits were too large to be explained by monocular vernier thresholds obtained for the same targets. In addition, fusion limits were not affected by large changes in target contrast, whereas vernier thresholds increased as contrast was decreased. The results of Experiment 1 also argued against interocular suppression as the cause of single vision, since vernier offsets that were visible when viewed monocularly were invisible under binocular viewing conditions. In Experiment 2, manual adjustment of disparities yielded fusion limits little different from those obtained with the forced-choice method of Experiment 1, demonstrating that it is possible to design adjustment methods for assessing fusion limits that are as sensitive as forced-choice methods. In Experiment 3, large reductions in target contrast, which have the effect of decreasing disparity sensitivity, did not alter fusion limits, disconfirming the idea that fusion limits estimated with discriminative procedures represent disparity-detection thresholds. In Experiment 4, disparities were adjusted until a just noticeable difference in grating contrast appeared. These disparities were larger than fusion limits, indicating that fusion limits did not represent a change in apparent contrast arising from disparity limitations of binocular summation. Together, the four experiments support the existence of binocular fusion as a unique category of sensory performance, disconfirm several nonfusional explanations of single vision, and support the use of criterion-free as well as adjustment methods in measuring fusion limits.
\end{abstract}

Although our two eyes provide two images of the world seen from slightly different directions, objects are generally experienced as single and lying in a direction that is intermediate to the two disparate views (Sheedy \& Fry, 1979). When physiological processes such as motor vergence and interocular suppression are factored out, this experience of single vision and averaging of visual direction may be looked upon as resulting from a sensory mechanism that blends or "fuses" the views given by the two eyes (Kertesz, 1972; Ono \& Barbeito, 1982; Panum, 1858; Sheedy \& Fry, 1979). The range of interocular disparities within which objects viewed with both eyes appear single is known as Panum's fusional area. The upper limit of this range of disparities may be termed the fusion limit, which has been characterized as the upper

This project was supported by National Eye Institute Training Grant 5T32-EYO7043 to Thomas Heckmann and Grant EYO3532 to Clifton M. Schor. Address correspondence to Thomas Heckmann, who is now at Human Performance Laboratory, Institute for Space and Terrestrial Science, York University, North York, Ontario M3J 1P3, Canada. disparity tolerance of the sensory mechanism for fusion (Mitchell, 1966). Consideration of the manner in which fusion limits have typically been measured, however, leaves the validity of such measurements open to question.

Fusion limits have typically been assessed by asking observers to adjust target disparities according to some phenomenal criterion for single vision or diplopia. The actual criteria employed have not always been reported (Kaufman \& Arditi, 1976). Also, a number of intermediate sensory states occur in the disparity range between unequivocal singleness and doubleness, such as binocular luster, binocular rivalry, and changes in apparent target width (Schor \& Heckmann, in press; Schor, Heckmann, \& Tyler, in press; Schor, Wood, \& Ogawa, 1984). A number of criteria related to different sensory states are therefore possible. This leads one to question whether a given disparity measurement obtained with an adjustment procedure truly reflects the disparity limits of a fusion mechanism, or, alternatively, the exercise of a judgment applied to some other disparity-dependent sensory factor (Arditi, 1986; Arditi \& Kaufman, 1978). When, however, freedom from judgment criteria has been achieved by use of methods that permit discrimination of 
disparate from nondisparate targets, the obtained results bring the existence of fusion itself into question.

Estimates of fusion limits are minimized when observers are forced to choose between two trial periods containing disparate and nondisparate targets. This finding has been obtained for torsional disparity (Arditi \& Kaufman, 1978; Kaufman \& Arditi, 1976), as well as for vertical disparity (Duwaer \& van den Brink, 1981, 1982). Such fusion limits are smaller than those obtained with even the most restrictive adjustment criterion, that of unequivocal single vision. Torsional and vertical fusion limits are even further reduced when the discriminative, criterionfree aspect of two-alternative forced-choice (2AFC) procedures is optimized by making a zero-disparity standard target available during disparate and nondisparate trial periods alike (Arditi \& Kaufman, 1978; Duwaer \& van den Brink, 1981, 1982; Kaufman \& Arditi, 1976). Torsional fusion limits obtained by Kaufman and Arditi (1976) were, in fact, small enough to be accounted for by monocular vernier sensitivity. Thus, if the minimum estimates of fusion limits provided by criterion-free procedures can be attributed to monocular vernier sensitivity, and if the larger fusion limits obtained with adjustment procedures can be attributed to "observers' adoption of lenient criteria in making such judgements" (Arditi, 1986, p. 23-33), fusion itself disappears under analysis as a unique category of sensory performance (Arditi, 1986; Arditi \& Kaufman, 1978; Kaufman \& Arditi, 1976). However, the experiments that have compared fusion limits to vernier thresholds may be of limited generality, since they were performed with line targets, which have a wide spatial band and contain high spatial frequencies. There is reason to believe that fusion limits and vernier thresholds obtained with narrow-band targets would give somewhat different results.

There is good evidence that luminance-domain spatial bandpass channels process disparities of magnitudes proportional to the spatial periods of peak channel sensitivities. For example, the widths of disparity-tuning curves for disparity-sensitive units in the cat cortex are positively correlated with their receptive field sizes (Ferster, 1981; Pettigrew, Nikara, \& Bishop, 1968). A psychophysical parallel is the occurrence of disparity-selective contrast adaptation effects in which the disparity magnitude for peak adaptation is proportional to the spatial period of the target (Felton, Richards, \& Smith, 1972). Such findings predict that fusion limits will decrease as the spatial frequency of narrow-band targets increases. This prediction has been confirmed, using an adjustment technique, for difference-of-Gaussian (DOG; Schor et al., 1984) and Cauchy-function luminance profiles (Schor et al., in press). In addition, Schor et al. (in press) found that vertical fusion limits for sinusoidal gratings obtained with a criterion-free procedure are similarly scaled to target spatial period. This spatial scaling of fusion limits is not simply a function of target size. Schor et al. (1984) found that fusion limits for a bright, square-pulsed bar remained as small as those obtained with the highest frequencies of DOG targets, in spite of large changes in bar width. This finding suggests that wide-band targets that contain high spatial frequencies, such as lines, dots, or bars, will ensure minimum fusion-limit estimates for any method employed. Furthermore, if the method itself is one that ensures minimum fusion-limit estimates, then no difference between vernier thresholds and fusion limits will be evident. The power of a test of differences between fusion limits and vernier thresholds might, however, be increased by using relatively low-frequency bandpass targets to increase the size of fusion limits.

The first purpose of the present study, therefore, was to explore whether vertical fusion limits for sinusoidal gratings collapse into monocular vernier thresholds, when both are estimated with the same criterion-free procedure. In Experiment 1, vertical fusion limits and monocular vernier thresholds for horizontal sinusoidal grating targets of $0.4-3.2$ cycles per degree (cpd) and $60 \%$ contrast were obtained with a 2 AFC procedure. The manipulation of disparity entailed opposite but equal vernier offsets in the two eyes. This permitted vernier thresholds to be directly compared with the monocular vernier offsets that corresponded to fusion-limit disparities. The method of disparity manipulation also enhanced the discriminative, or criterion-free, aspect of the task by ensuring that a zerodisparity stimulus was available for comparison with the variable-disparity stimulus during all target presentations. As an additional test of the dependence of fusion limits on vernier sensitivity, monocular vernier thresholds were repeated for one target $(0.8 \mathrm{cpd})$ at a lower contrast (15\%). Vernier thresholds for sinusoidal gratings increase with decreasing contrast according to a power law having an exponent of about -0.8 (Bradley \& Skottun, 1987). Since fusion limits for sinusoids measured with the present criterion-free method are, however, unresponsive to large changes in contrast (Schor et al., in press), we reasoned that vernier thresholds under the present experimental conditions must also be unresponsive to contrast in order to account for fusion limits.

A second purpose of the present work was to test whether small fusion limits are afforded by criterion-free methods primarily because of the availability of a zerodisparity comparison stimulus, independent of specific psychophysical procedures. Experiment 2 thus employed the same discriminative target as Experiment 1, but fusion limits were obtained by manual adjustment of disparity.

The third purpose was to evaluate whether fusion limits obtained with criterion-free procedures might owe to sensory processes other than fusion that operate in the same disparity range. Alternative processes considered were interocular suppression, a minimum disparity sensitivity, and binocular contrast summation.

Regarding interocular suppression, Kaufman and Arditi (1976) suggested that fusion limits may actually represent upper disparity limits for interocular suppression, in which case single vision occurs because only the view of one eye is seen. The comparison of monocular vernier off- 
sets in Experiment 1 served to test this hypothesis: If the opposite vernier offsets in each of the two eyes at a given fusion limit were larger than the monocular vernier threshold for the same target, then single vision in the range of vernier offsets between the vernier threshold and the vernier offset corresponding to the fusion limit could not be attributed to experiencing the view of only one eye, that is, to interocular suppression.

Duwaer and van den Brink $(1981,1982)$ attributed the small disparity-discrimination thresholds that they obtained with forced-choice measures to minimum disparity sensitivity. In this case, disparity estimates ought to depend strongly on contrast, in view of evidence from contrast adaptation experiments that disparity is processed by contrast-sensitive spatial bandpass mechanisms (Blakemore \& Hague, 1972; Felton et al., 1972). This view is further supported by the fact that stereothresholds decrease as a power function of contrast (Halpern \& Blake, in press; Heckmann \& Schor, in press; Legge \& Gu, in press). Furthermore, the less-than-unity slope of this power function is predicted by the brightness compression properties of luminance-domain bandpass channels (Halpern \& Blake, in press). Yet vertical fusion limits for sinusoidal gratings obtained with a criterion-free procedure resist as much as a 16-fold change in target contrast, suggesting that minimum disparity sensitivity is not involved in determining fusion limits (Schor et al., in press). Experiment 3 replicated this contrast manipulation for the fusion limits obtained with the adjustment procedure of Experiment 2.

Experiment 4 tested whether fusion limit measurements might actually reflect a just noticeable difference (jnd) in apparent contrast with increasing stimulus disparity. This possibility is suggested by evidence that disparity influences binocular contrast summation. Contrast thresholds for dichoptically viewed sinusoidal gratings (Blakemore \& Hague, 1972) or narrow-bandpass targets (Rose, Blake, \& Halpern, 1988) become elevated as their disparities are increased. Observers in Experiment 4 adjusted the disparities of sinusoidal targets until they appeared just different in contrast from their zero-disparity neighbors, and these settings were compared with fusion limits.

\section{METHODS}

Four experiments were conducted. In Experiment 1, a 2AFC staircase procedure was used to estimate fusion limits and monocular vernier thresholds in the vertical meridian. In Experiment 2, target disparities were adjusted to the greatest magnitudes at which their appearances matched those of adjacent zero-disparity standard targets. Experiment 3 obtained maximum-disparity matches using the same adjustment procedure as Experiment 2, but for targets of reduced contrast. In Experiment 4, disparities were adjusted to produce jnds in apparent contrast between disparate targets and adjacent zero-disparity standard targets.

\section{Binocular Targets}

Horizontal sinusoidal grating targets were used in all four experiments. All measurements were obtained with vertical rather than horizontal target offsets to eliminate stereoscopic depth as a cue to disparity. Since fusion limits are generally smaller in the vertical than in the horizontal meridian (Schor et al., in press; Schor \& Tyler, 1981; Schor et al., 1984; Tyler, 1975), use of vertical disparity helped to minimize the likelihood of false fusion to adjacent grating cycles (the wallpaper illusion). It was anticipated that a 2 AFC procedure would, in addition, minimize fusion limits, and further decrease the likelihood of false fusion of grating patterns.

Gratings were generated by digital-to-analog conversion of lookup tables stored in EPROMS. Voltage analogs of these one-dimensional functions controlled the $z$-axis luminance modulation of two Tektronix model 608 oscilloscopes, viewed haploscopically at a distance of $57 \mathrm{~cm}$. Mean luminance in all experiments was $55 \mathrm{~cd} / \mathrm{m}^{2}$. Contrast of gratings was controlled by analog modulation of the z-axis signal.

\section{Psychophysical Methods}

Observers haploscopically viewed a $5^{\circ}$ circular bipartite field divided by a vertical $10^{\prime}$ of arc wide strip of opaque tape (Figure 1). Both halves of the field contained the horizontally oriented sinusoidal pattern. The right half of the field always subtended zero disparity. The left half of the field either subtended zero disparity or was shifted upward in front of the left eye and downward in front of the right eye. The use of this bipartite-field display, in which opposite vernier offsets in the two eyes were used to produce disparities, allowed direct comparison between threshold monocular vernier offsets and monocular vernier offsets corresponding to fusion-limit disparities. Analog electronic procedures were employed to achieve the vertical bipartite division of oscilloscope displays and to shift the left halves of the two displays with respect to their right halves. A line of three binocular fixation marks appeared in the left half of the haploscopic display, centered on the vertical midline. Nonius lines appeared in the left and right halves of the display, flanking the central fixation mark.

Each staircase began with a distinctly diplopic vertical disparity in the left half of the field in one interval of the trial, and zero disparity in the other interval. In each presentation, contrast was driven upward with a ramp-shaped signal for $750 \mathrm{msec}$ from zero to maximum contrast, remained constant for $750 \mathrm{msec}$, and was then driven downward with a ramp-shaped signal to zero contrast over another $750 \mathrm{msec}$. The gradual increase and decrease in target contrast avoided apparent motion between different target offsets as a cue to correct trial period. Observers pressed buttons to indicate the interval in which the two hemifields of the bipartite display looked

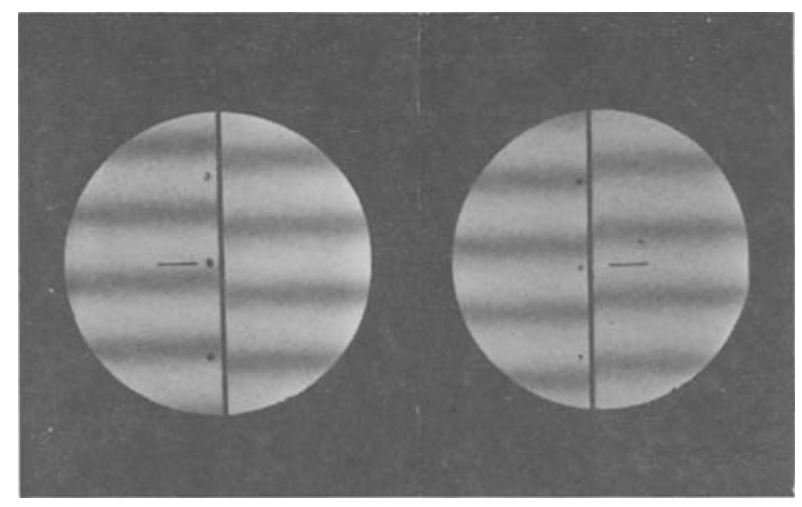

Figure 1. Stimulus display for obtaining estimates of vertical fusion limits. The two displays were viewed haploscopically. The right half of the bipartite field remained always at zero disparity. Vertical disparity was manipulated in the left half of the display by moving the targets viewed by the left and right eyes in opposite vertical directions. 
different. Incorrect choices were signaled by a distinctive tone. The staircase procedure converged on a threshold criterion of .794 after five reversals by a "three-correct, one-incorrect"' sequencing of disparity magnitude changes (Levitt, 1971). Thresholds were averaged over four separate staircase determinations.

Observers were required to maintain binocular fixation throughout each staircase session. Fixation marks and nonius lines assured control of disjunctive eye movements to within $1^{\prime}$ of arc (Schor et al., 1984). Placement of fixation marks in the left half of the display provided some balance to the vergence stimulus afforded by the zero-disparity grating in the right half of the display.

For determination of monocular vernier thresholds, the $2 \mathrm{AFC}$ procedure was the same as that used for estimating fusion limits, except that one eye remained covered. Fusion limit and vernier threshold data were taken after a stable level of performance had been obtained with repeated sessions.

Experiment 2 employed the same bipartite display as Experiment 1 . To estimate fusion limits by adjustment, observers controlled vertical disparities in the left side of the display by means of a potentiometer. Target contrast was continuously turned on and off in the same manner as in Experiment 1, to avoid contamination of fusion limit determinations by the buildup of suppression and rivalry effects. Since the presence of the standard target afforded a true discrimination task, arguably rendering it criterion-free, two criterion conditions were employed to assess any residual effects of criteria upon adjustments. In the first, or same-width (SW), condition, disparity was increased in each of five trials until dark bands on the left half of the display appeared to be just noticeably wider than those on the zero-disparity, right side of the display. In five other trials, disparity was decreased until left-side bands just appeared to be the same width as right-side bands. The fusion limit was averaged over the $\mathbf{1 0}$ trials. In the second, or no-difference (ND), criterion condition, disparity was increased until the left half of the display appeared just different from the right half, or was decreased until the two halves appeared just the same.

Fusion limits obtained under the reduced-contrast conditions of Experiment 3 employed the same adjustment method as in Experiment 2, but used only the SW criterion. Experiment 4 utilized the same display and method of adjustment as in Experiments 2 and 3 , except that the judgment criterion was the appearance (increasingdisparity trials) or disappearance (decreasing-disparity trials) of a difference in apparent peak-to-trough contrast across the disparate and nondisparate halves of the display.

\section{Observers}

The observers were the two authors (T.H. and C.S.). Both were well experienced in psychophysical experiments, and had mild myopia and no anomalies of binocular fusion or stereopsis as defined by standard clinical tests. The observers wore their refractive corrections and viewed targets with natural pupils. The observers were well practiced in maintaining good binocular fixation, as described elsewhere (Heckmann \& Schor, in press).

\section{EXPERIMENT 1}

\section{Fusion Limits and Monocular Vernier Thresholds Estimated with a Forced-Choice Discrimination Procedure}

Fusion limits and monocular vernier thresholds were determined with the 2AFC procedure for sinusoidal gratings having spatial frequencies of $0.4,0.8,1.6$, and $3.2 \mathrm{cpd}$. These spatial frequencies span the frequency range over which fusion limits decrease to minimum values with increasing spatial frequency of DOG targets (Schor et al., 1984). Since only two cycles of the lowest spatial frequency $(0.4 \mathrm{cpd})$ were visible within a $5^{\circ}$ aperture, the spatial frequency setting was doubled and the distance to the display was halved, so that four cycles of the lowest frequency were viewed in a $10^{\circ}$ field. Target contrast was $60 \%$, except for the $15 \%$ contrast employed to test the effect of contrast on vernier sensitivity at $0.8 \mathrm{cpd}$.

\section{Results}

Vertical fusion limits are plotted against spatial frequencies of sinusoidal targets in Figure 2 (circles). Fusion limits in Figure 2 are expressed as minutes of arc of monocular vernier offset present to one eye at the fusion limit. This convention provided for direct comparison of monocular vernier thresholds to fusion limits. It also gave a convenient test of the hypothesis that fusion limits based on discrimination of disparate and nondisparate targets actually represent monocular vernier sensitivities.
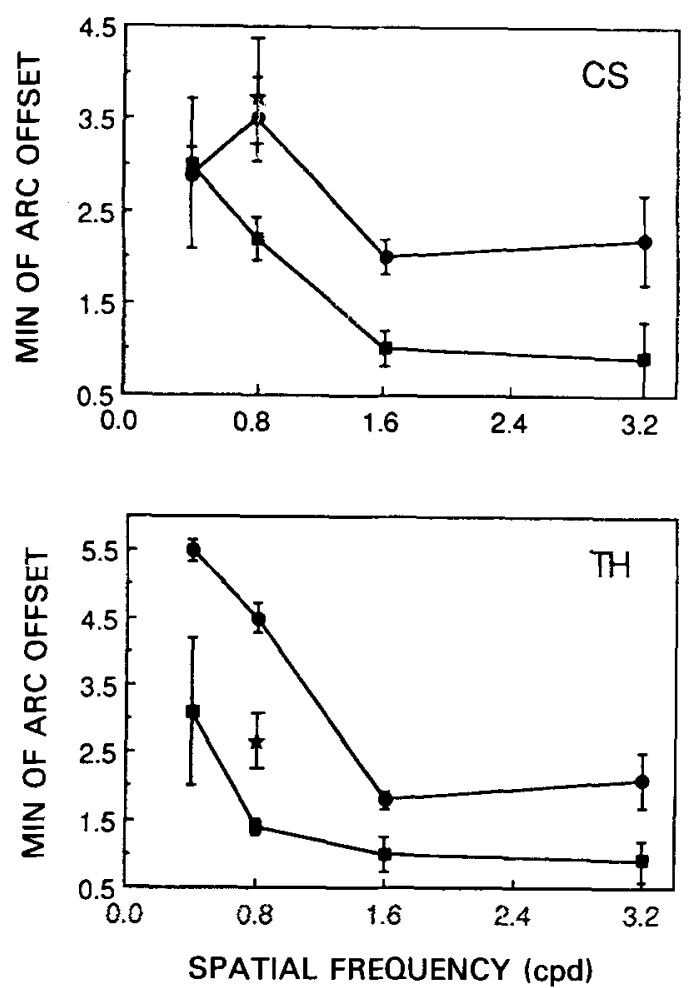

Figure 2. Vertical vernier offsets in each eye which were present at fusion-limit disparities (circles) and monocular vernier thresholds (squares) for two observers. Both measurements were obtained with a two-alternative forced-choice discrimination procedure and sinusoidal grating targets of 0.4-3.2 cycles per degree (cpd). Target contrast was $60 \%$. Fusion limits decrease with increasing spatial frequency, in particular between 0.8 and $1.6 \mathrm{cpd}$. Monocular vernier thresholds also decrease with increasing spatial frequency, but, with the exception of Observer C.S. at $0.4 \mathrm{cpd}$, are too small to account for discrimination between disparate and nondisparate targets in the binocular case. Vernier thresholds measured at $0.8 \mathrm{cpd}$ and $15 \%$ contrast (stars) are $1.5 X-2 X$ greater than those measured at $60 \%$ contrast. A comparable contrast manipulation does not influence fusion limits (Schor et al., 1989; Figure 4, below). Error bars represent $\pm 1 S D$. 
Fusion limits decrease with increasing spatial frequency, and level off between 1.6 and $3.2 \mathrm{cpd}$. This finding is consistent with the behavior of fusion limits obtained with bandpass targets and a method of adjustment that employed a subjective criterion for single vision (Schor et al., in press; Schor et al., 1984). The chief difference in the present results is a twofold to fivefold reduction in the absolute magnitude of fusion limits, with the largest reduction occurring at the lowest spatial frequency. There was no evidence in this experiment for disparity hysteresis (e.g., Fender \& Julesz, 1967), which would have been reflected in a consistent tendency for the number of steps associated with increasing-disparity staircases to exceed the number associated with decreasing-disparity staircases. It should be noted that chance alone would have favored apparent hysteresis, since the staircase procedure required only one mistake for disparity to increase a step, but three correct discriminations for it to decrease a step.

Vertical monocular vernier thresholds are plotted against spatial frequency in Figure 2 (squares). As with fusion limits, vernier thresholds decrease with increasing spatial frequency of sinusoidal targets. Vernier thresholds are, with one exception (Observer C.S. at $0.4 \mathrm{cpd}$ ), less than the corresponding monocular vernier offsets present at fusion limits. But for the exception noted, however, there is no overlap for individual data points contributing to the means of the two measures.

The reduced-contrast vernier threshold estimates $(0.8 \mathrm{cpd}$, $15 \%$ contrast) are represented by the large open circles in Figure 2. The elevated vernier thresholds are consistent with the more thorough data of Bradley and Skottun (1987), and represent an effect of contrast that is not shared by fusion limits measured with the same methods as those employed by Schor et al. (in press) and in Experiment 3 (below).

\section{EXPERIMENT 2}

\section{A Criterion-Free Method of Adjustment for Fusion Limits}

Experiment 2 sought to determine whether the conservative estimates of fusion limits obtained in Experiment 1 were dependent on the $2 \mathrm{AFC}$ staircase procedure, or on the bipartite-field display that permitted simultaneous comparison of variable-disparity and zero-disparity targets. Equivalent results for 2AFC and adjustment methods using the bipartite-field display would imply that the same sensory performance was being assessed with the two procedures. One might then dispense with the more timeconsuming 2AFC method. Procedures for Experiment 2 are described under Methods, above. The same sinusoidal grating targets were employed as in Experiment 1 $(0.4,0.8,1.6$, and $3.2 \mathrm{cpd}, 60 \%$ contrast $)$.

\section{Results}

Fusion limits estimated with the adjustment method and the two judgment criteria, SW (same width of dark bars across the bipartite field; dashed-and-dotted lines) and ND
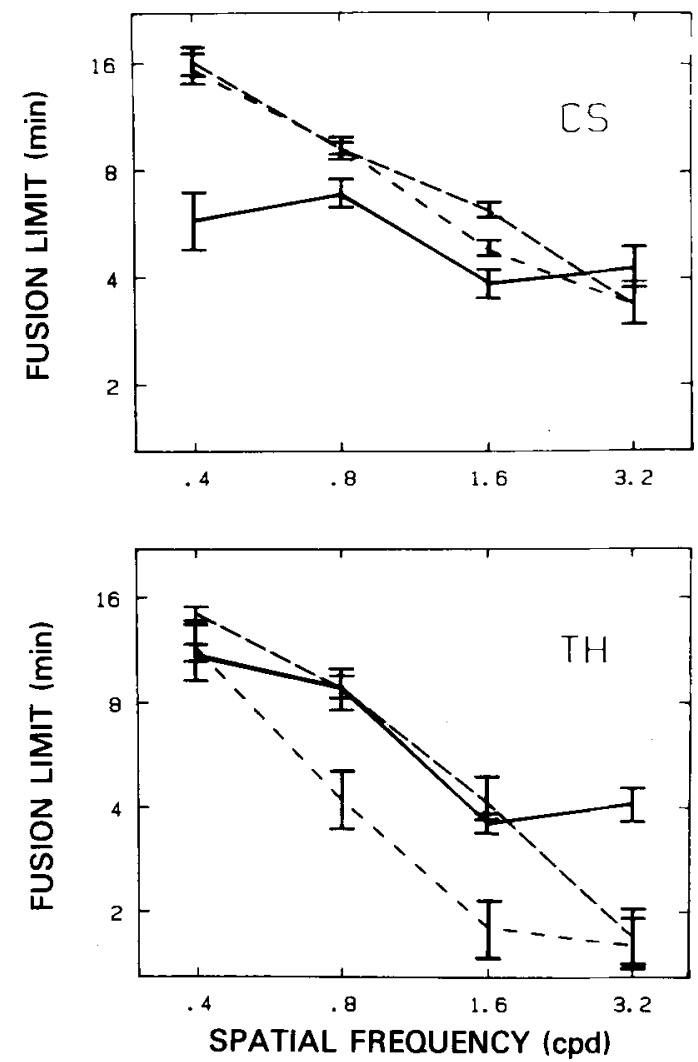

Figure 3. Vertical fusion limits obtained through adjusting the variable-disparity target to the greatest disparity that gave a match to the appearance of the zero-disparity standard target. Dashed and dashed-and-dotted lines, respectively, represent fusion limits obtained with the no-difference and same-width discrimination criteria. Fusion limits obtained with the two-alternative forced-choice (2AFC) procedure (solid lines) are presented for the sake of comparison. With the exception of the lowest spatial frequency in Observer C.S. and the highest spatial frequency in Observer T.H., fusion limits obtained with the same-width criterion are comparable to those obtained with the $2 A F C$ method. Error bars represent $\pm 1 S E$.

(no difference between sides of the bipartite field; dashed lines), are plotted against spatial frequency in Figure 3, as radii of Panum's fusional area in minutes of arc. Fusion limits obtained in Experiment 1 with the 2AFC method are plotted for comparison (solid lines). As with the 2AFC method, fusion limits decrease with increasing target spatial frequency. In addition, the absolute magnitudes of fusion limits are similar to those obtained with the 2AFC method, being about half those obtained by Schor et al. (1984) with a method of adjustment and a subjective criterion for single vision. Inspection of individual increasing-disparity and decreasing-disparity adjustments yielded no evidence for disparity hysteresis.

The only consistent difference between fusion limits obtained with 2AFC and adjustment methods is that fusion limits obtained with adjustment tend to decrease between 1.6 and $3.2 \mathrm{cpd}$, rather than leveling off between these two frequencies. Other differences between results obtained with the two methods are generally small and idio- 
syncratic. For Observer C.S., the fusion limit at $0.4 \mathrm{cpd}$ is nearly tripled in the adjustment compared with the $2 \mathrm{AFC}$ data. Yet fusion limits obtained with adjustment are still about half those estimated for the same observer by Schor et al. (1984) with a criterion-dependent method. Except at $0.4 \mathrm{cpd}$, fusion limits for Observer C.S. obtained by adjustment are no more than about $2^{\prime}$ different in disparity from those obtained with the criterion-free method of Experiment 1. Also, there is almost no difference owing to judgment criterion in this observer. For Observer T.H., there is little difference between fusion limits estimated with the 2AFC method and those obtained by adjustment employing the $\mathrm{SW}$ criterion, except at 3.2 cpd. Fusion limits obtained with adjustment and the ND criterion are about half those obtained with the SW criterion at $0.8^{\circ}$ and $1.6^{\circ}$. The two criteria nonetheless give similar data at 0.4 and $3.2 \mathrm{cpd}$. Although fusion limits for Observer T.H. at $3.2 \mathrm{cpd}$ are small enough to be accounted for by monocular vernier sensitivity, this is true for none of the other fusion limits in this experiment.

\section{EXPERIMENT 3}

\section{Fusion Limits at Low Contrast}

When fusion limits are measured with the 2AFC procedure employed in Experiment 1, they remain constant across a twofold decrease in contrast at $0.4 \mathrm{cpd}$, and as much as a 16-fold decrease in contrast at $1.6 \mathrm{cpd}$ and higher (Schor et al., in press). A finding that fusion limits obtained with the adjustment method of Experiment 2 are similarly unresponsive to large changes in contrast would provide further assurance that the adjustment method assesses the same sensory performance as the 2AFC method, and that, according to arguments outlined above, the present estimates of fusion limits do not actually represent minimum disparity sensitivities. Fusion limits were measured with the adjustment method at relatively low contrasts, using the SW criterion. The SW criterion was chosen because, overall, it gave fusion limits in Experiment 2 that were more consistent with the 2AFC results of Experiment 1 than were fusion limits obtained with the ND criterion. The contrast levels chosen were $30 \%, 15 \%, 7.5 \%$, and $7.5 \%$, respectively, for $0.4,0.8$, 1.6 , and $3.2 \mathrm{cpd}$. These approximated the lower contrast levels used by Schor et al. (in press) in estimating fusion limits with the 2AFC method, and were the lowest contrasts at which fusion limits could be reliably obtained with the adjustment technique.

\section{Results}

Figure 4 compares fusion limits obtained with the SW criterion in Experiment 2 at $60 \%$ contrast (dashed-anddotted lines) with those obtained with the same criterion at lower contrast levels (double-dotted-and-dashed lines). The curves describing the dependence of fusion limits on spatial frequency are virtually superimposable for high and low contrast.
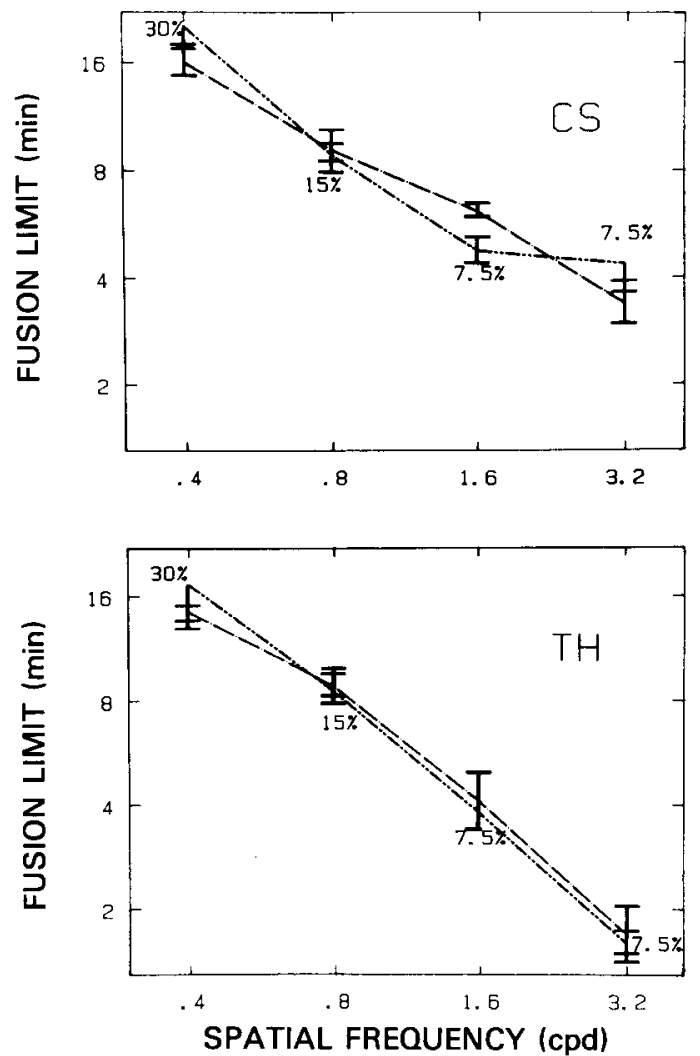

Figure 4. Vertical fusion limits obtained with the adjustment method and the same-width criterion for different levels of contrast. The dashed-and-dotted lines represent fusion limits obtained at $60 \%$ contrast. The double-dotted-and-dashed lines represent the lowest contrast levels at which fusion limits could be reliably obtained. These contrasts are noted in the figure at each datum point. While fusion limits decrease with increasing spatial frequency, wide variations in suprathreshold contrast do not affect fusion limits. Error bars represent $\pm 1 S E$.

\section{EXPERIMENT 4}

\section{Disparities that Produce jnds in Apparent Contrast}

Fusion limits measured with the 2AFC procedure of Experiment 1 were compared with disparities adjusted to produce a jnd in apparent contrast between the two halves of the bipartite display. Sinusoidal gratings of 0.4 and $1.6 \mathrm{cpd}$ were employed. Data were obtained at $60 \%$ contrast for both spatial frequencies. Fusion limits and disparities for apparent contrast jnds were also obtained at $30 \%$ and $7.5 \%$ contrast, respectively, for 0.4 and $1.6 \mathrm{cpd}$. The lower contrast at each frequency was near the lower limit of a contrast range across which there is no change in fusion limits (Schor et al., in press).

\section{Results}

The histograms of Figure 5 illustrate that fusion limits obtained with the 2AFC method of Experiment 1 are smaller than the disparities at which jnds in apparent contrast occur. The maximum fusion limit at $0.4 \mathrm{cpd}$ is $12^{\prime}$ 

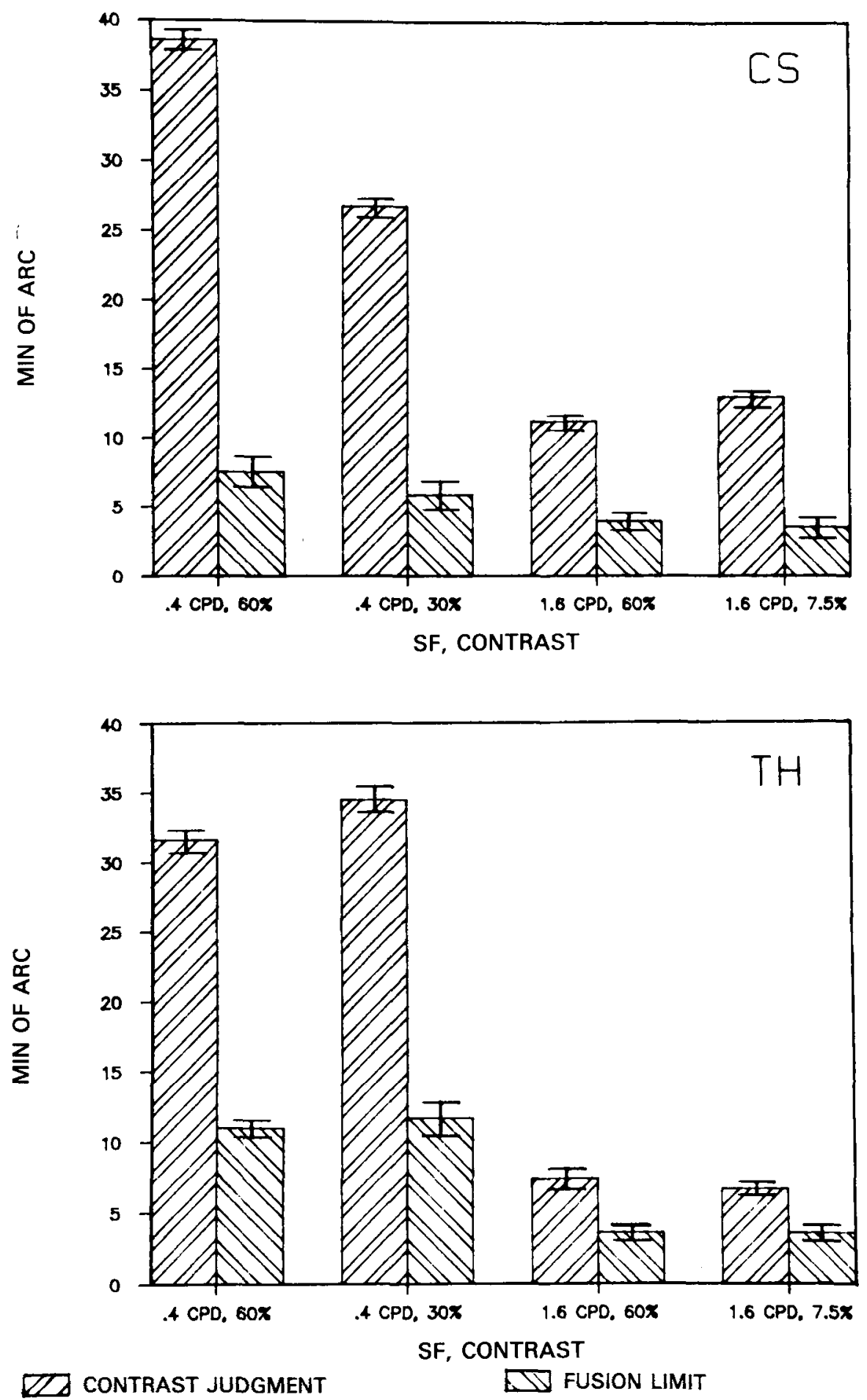

Figure 5. Disparity limits necessary to obtain a just noticeable difference (jnd) in apparent contrast between disparate and nondisparate targets (left-hand member of each pair of histograms) are compared at two spatial frequencies and two levels of contrast to fusion limits obtained with the two-alternative forced-choice method (right-hand member of each pair of histograms). Disparity limits for jnds in apparent contrast are too large to account for fusion limits. Error bars represent $\pm 1 S E$. 
of arc. This disparity translates into a $28.8^{\circ}$ interocular shift in the phase of the target period $\left(28.8^{\circ}\right.$ phase disparity). The maximum and minimum disparities for apparent contrast jnds at $0.4 \mathrm{cpd}$ are $27^{\prime}$ and $38^{\prime}$ of arc, representing, respectively, $64.8^{\circ}$ and $91.2^{\circ}$ phase disparity. Fusion limits at $1.6 \mathrm{cpd}$ are about $4^{\prime}$ of arc, or $38.4^{\circ}$ phase disparity. Disparity limits for apparent contrast jnds at $1.6 \mathrm{cpd}$ range between $7^{\prime}$ and $13^{\prime}$ of arc, or $67.2^{\circ}$ and $124.8^{\circ}$ phase disparity. If fusion limits from Experiments 2 and 3, obtained with a method of adjustment, are substituted for the $2 \mathrm{AFC}$ data, the only difference is an elevation in the maximum fusion limit at $0.4 \mathrm{cpd}$ from 12' to $16^{\prime}$ of arc, or to $38.4^{\circ}$ phase disparity. This is still much less than the phase disparity for a jnd in apparent contrast.

\section{DISCUSSION}

\section{Subjective Criteria Versus \\ Criterion-Free Methods}

Vertical fusion limits were estimated with a criterionfree $2 \mathrm{AFC}$ method. In agreement with previous findings (Arditi \& Kaufman, 1978; Duwaer \& van den Brink, 1981, 1982; Kaufman \& Arditi, 1976), the criterion-free method gave fusion limits far smaller than those obtained with a criterion-dependent adjustment method (e.g., Schor et al., in press; Schor et al., 1984). So-called criterionfree methods alone do not, however, guarantee against the use of different subjective criteria. Different subjective criteria may, in fact, be deliberately imposed on such methods, and produce different results. As a case in point, Duwaer and van den Brink (1981) obtained larger vertical fusion limits when the criterion of unequivocal double vision was used, instead of unequivocal single vision, to discriminate successive trial periods.

In addition to differences in subjective criteria, the use of categorically different perceptual responses can influence disparity discriminations made with forced-choice psychophysics. Duwaer and van den Brink (1981) characterized thresholds for discrimination between disparate and nondisparate targets as minimum disparity sensitivities, regardless of meridian, and found smaller disparity thresholds in the horizontal than in the vertical meridian. Although perceived singleness or doubleness was used to discriminate vertical disparities, perceived depth was explicitly employed for discrimination of horizontal disparities. Clearly, an upper disparity tolerance does not explain their horizontal-meridian results. Consistent application of a singleness or doubleness discrimination across meridia, however, might have led these authors to quite different results. Assuming that an upper disparity tolerance for fusion controls forced-choice discriminations of doubleness or singleness, larger rather than smaller discrimination thresholds would be predicted in the horizontal than in the vertical meridian. This prediction follows from the fact that, when one criterion is employed for disparity adjustments in both meridia, fusion limits are consistently larger in the horizontal than in the vertical meridian (Schor et al., in press; Schor \& Tyler, 1981; Schor et al., 1984; Tyler, 1975).
If the magnitudes of fusion limits obtained with adjustment procedures can be attributed to the exercise of lenient subjective criteria for single vision rather than to some underlying sensory process, then one would not expect such a consistent effect of meridian. Additionally, if fusion limits obtained with different measurement techniques differ primarily in absolute magnitude, but maintain the same proportional relationship to some stimulus magnitude, then it is likely that the different test procedures evaluate the same sensory process. Accordingly, when Schor et al. (in press) plotted fusion limits obtained with the present $2 \mathrm{AFC}$ procedure against spatial frequency, the resulting slope was similar to that plotted for fusion limits obtained with a method of adjustment utilizing a subjective criterion for single vision (Schor et al., 1984), although the latter fusion limits had greater absolute magnitudes.

\section{A Criterion-Free Method of Adjustment}

Even within an unequivocally single range of disparities, fusion limits are further reduced by the presence of a zero-disparity standard stimulus during both periods of a temporal 2AFC procedure (Arditi \& Kaufman, 1978; Duwaer \& van den Brink, 1981, 1982; Kaufman \& Arditi, 1976). Zero-disparity standard targets were therefore employed in the 2AFC procedure of Experiment 1 to minimize fusion limits. In addition, fusion limits were obtained in Experiments 2 and 3 with an adjustment procedure, but with the same bipartite-field display as in Experiment 1 , which provided zero-disparity standard targets during disparity adjustments. The spatial frequency dependence of fusion limits (Schor et al., in press; Schor et al., 1984) was again replicated, as was the absence of dependence on suprathreshold target contrast (Schor et al., in press). Finally, fusion limits measured by adjustment in Experiment 2 were not only smaller than those obtained for bandpass targets by adjustment according to a subjective singleness criterion (Schor et al., in press; Schor et al., 1984), but were equivalent in magnitude to those obtained with the 2AFC procedure of Experiment 1. It therefore seems that the reduction in fusion limits that is obtained with criterion-free procedures owes more to the availability of a zero-disparity standard target than it does to a specific psychophysical procedure.

The small differences in fusion limits obtained between the $2 \mathrm{AFC}$ procedure of Experiment 1 and the adjustment method of Experiment 2 most likely represent differences between two response tasks. For example, the buttonpushing used in the 2AFC procedure does not change disparity directly, whereas the knob-twisting response used for adjustment not only does so, but provides proprioceptive feedback that is correlated with disparity. Residual judgment bias may also have been a factor. The lower fusion limits in the case of adjustment at the highest frequency may occur because trained observers expect small fusion limits at high spatial frequencies. In view of the small differences in results obtained with the two procedures, however, it is questionable whether timeconsuming and technically elaborate forced-choice 
methods should be chosen for estimating fusion limits, even if minimum estimates are desired.

\section{Alternative Sensory Explanations of Fusion Limits}

In the present study, minimum estimates of fusion limits were desired in order to test whether fusion limits might be accounted for by factors other than binocular sensory fusion. Specifically, these alternatives included (1) monocular vernier sensitivity, (2) an upper limit to a disparity range for interocular suppression, (3) a minimum threshold for disparity sensitivity, and (4) a disparity limit at which a jnd in apparent target contrast occurs because of a decline in binocular contrast summation with increasing disparity.

Monocular vernier sensitivity. The idea that the fusion limit is an artifact of monocular vernier sensitivity is consistent with good agreement between the two measures at extrafoveal locations (Le Grand, 1967, p. 209). This evaluation is supported by forced-choice threshold values for torsional disparity discrimination, which are similar to those obtained for monocular target rotations (Arditi \& Kaufman, 1978; Kaufman and Arditi, 1976). The case of vertical disparity is somewhat different. Thresholds of only a few seconds of arc are typically reported for vernier displacements of adjacent targets at the fovea (see reviews by Graham, 1965; Howard, 1982; Le Grand, 1967). Westheimer and McKee (1977, Figure 3) reported monocular vernier thresholds in the vertical meridian of about 6" of arc for targets composed of adjacent horizontal lines having a $3^{\prime}$ of arc gap between them. Their target was thus similar to that used by Duwaer and van den Brink (1981) for discrimination of vertically disparate from nondisparate horizontal lines. Assuming that fusion limits actually reflect monocular vernier sensitivity, the threshold figure of Westheimer and McKee (6" of arc) predicts a maximum disparity discrimination threshold of 12 " of arc. This is an order of magnitude smaller than the approximately $2^{\prime}$ of arc vertical disparity threshold obtained by Duwaer and van den Brink. It is therefore unlikely that their vertical disparity discriminations represented monocular vernier sensitivities. The direct comparison of monocular vernier thresholds to fusion limits in the present study additionally disconfirms the origin of fusion limits in monocular displacement sensitivity. Finally, the fact that fusion limits are insensitive to manipulations of contrast that strongly influence vernier thresholds argues that fundamentally different mechanisms underlie the two measures.

Interocular suppression. In Experiment 1, suprathreshold monocular vernier offsets corresponding to disparities less than fusion limits were not visible during binocular viewing. Although averaging of visual direction, or allelotropia, may be independent of single vision (Rose \& Blake, 1988), invisibility of monocular vernier offsets during binocular viewing suggests that a binocular mechanism for directional averaging masks suprathreshold vernier offsets within the presently estimated disparity range for single vision. Apart from the fact that stimulus durations ( $750 \mathrm{msec}$ at full contrast) were too brief for interocular suppression and rivalry effects to build up, the binocular masking of suprathreshold monocular vernier offsets disconfirms interocular suppression as the cause of single vision in the present experiments. If interocular suppression were involved, then one would have been able to discriminate the disparate from the nondisparate side of the grating display on the basis of an unsuppressed vernier offset, and the curves of Figure 2 would be superimposed.

Absolute disparity sensitivity. The fact that fusion limits are not influenced by large reductions in stimulus contrast is of particular relevance to the suggestion by Duwaer and van den Brink $(1981,1982)$ that these limits represent thresholds for disparity detection. Duwaer and van den Brink (1981) obtained horizontal-meridian depthdetection thresholds of $0.2^{\prime}-0.3^{\prime}$ of arc, an order of magnitude less than thresholds obtained in the same study for discrimination of vertically disparate and nondisparate lines. Similarly, stereothresholds 10 to 20 times smaller than the fusion limits estimated in the present experiments have been obtained with the same sinusoidal grating targets (Heckmann \& Schor, in press). Yet, unlike fusion limits measured with discriminative procedures, stereothresholds decrease steadily as target contrast is increased (Halpern \& Blake, in press; Heckmann \& Schor, in press; Legge $\& \mathrm{Gu}$, in press). If the data for both meridia represent disparity sensitivity, therefore, the contrast-independent mechanism underlying disparity sensitivity in the vertical meridian must differ in some fundamental manner from the contrast-dependent mechanism that governs disparity sensitivity in the horizontal meridian. Alternatively, differences in the effects of contrast may reflect fundamental differences between mechanisms underlying fusion limits and stereopsis, rather than meridional differences in disparity sensitivity. Schor et al. (in press) provide a more detailed discussion of contrast-dependent and contrast-independent disparity effects.

Disparity for a jnd in apparent contrast. Contrast thresholds are elevated in proportion to increasing disparity of narrow spatial-band targets, indicating that binocular summation depends on the relative interocular phase at which targets are presented (Blakemore \& Hague, 1972; Rose et al., 1988). Although effects of disparity on binocular summation at suprathreshold contrast levels have not been reported, the threshold-level findings suggest that a jnd in apparent contrast with increasing target disparity could be a factor contributing to fusion limit estimates.

Disparities at which apparent-contrast jnds occurred in Experiment 4 are consistent with an interocular target phase shift of about $90^{\circ}$. This corresponds closely to the interocular phase shift represented by fusion limits obtained with bandpass targets and a criterion-dependent adjustment method (Schor et al., in press; Schor et al., 1984). Fusion limits obtained with discriminative proce- 
dures in Experiments 1-3 are nonetheless too small to be explained by the disparity-dependent apparent-contrast jnd. Thus, even though there is evidence that disparitydependent binocular contrast summation occurs in spatial channels that mediate fusion (Rose et al., 1988), the fusion limits measured in the present study do not appear to derive from an effect of binocular summation on the perception of contrast.

\section{Conclusions}

First, it is unlikely that nonfusional accounts of fusion limits can explain the upper disparity limits for single vision obtained in the present experiments: Fusion limits for sinusoidal gratings are insensitive to changes in contrast that strongly influence vernier sensitivity. Monocular vernier sensitivity cannot therefore explain the small fusion limits obtained with criterion-free methods. Fusion limits are also simply too large to be accounted for by monocular vernier thresholds measured at the same target contrast. Suprathreshold monocular vernier displacements are therefore masked by a binocular directionalaveraging mechanism, and single vision in the present experiments does not result from interocular suppression. The present results also question whether minimum disparity sensitivity or a change in apparent contrast with increasing disparity can account for fusion limits. Barring additional contending explanations, single vision within the present fusion limits must be attributed to a sensory fusion mechanism in the classical sense.

Second, a binocular sensory fusion mechanism is probably the sensory basis for at least some fusion limits measured with criterion-dependent procedures: The similarity in spatial scaling for fusion limits estimated with criterion-free and criterion-dependent methods argues for a common underlying mechanism. Therefore, since the sensory basis for fusion limits estimated with criterionfree procedures is probably fusion, its influence is not necessarily restricted to the small disparity range obtained with criterion-free procedures.

Third, an adjustment procedure that permits comparison of disparate and nondisparate targets produces fusion limits commensurate with the minimum estimates afforded by a forced-choice method. This finding supports the use of appropriately designed adjustment procedures in measuring fusion limits.

\section{REFERENCES}

ARDITI, A. (1986). Binocular vision. In K. R. Boff, L. Kaufman, \& J. P. Thomas (Eds.), Handbook of perception and human performance: Volume I. Sensory processes and perception (chap. 23). New York: Wiley.

Arditi, A., \& Kaufman, L. L. (1978). Singleness of vision and the initial appearance of binocular disparity. Vision Research, 18, 117-120.

BLAKEMORE, C., HAGUE, B. (1972). Evidence for disparity detecting neurones in the human visual system. Journal of Physiology (London), 225, 437-455.
Bradley, A., \& Skottun, B. C. (1987). Effects of contrast and spatial frequency on vernier acuity. Vision Research, 27, 1817-1824.

DUWAER, A. L., \& VAN DEN BRINK, G. (1981). What is the diplopia threshold? Perception \& Psychophysics, 29, 295-309.

DUWAER, A. L., \& VAN DEN BRINK, G. (1982). The effect of presentation time on detection and diplopia thresholds for vertical disparities. Vision Research, 22, 183-189.

Felton, T. B., RichaRds, W., \& SMith, R. A., JR. (1972). Disparity processing of spatial frequencies in man. Journal of Physiology (London), 225, 349-362.

Fender, D. H., \& JULEsz, B. (1967). Extension of Panum's fusional area in binocularly stabilized vision. Journal of the Optical Society of America, 57, 819-830.

FERSTER, D. (1981). A comparison of binocular depth mechanisms in areas 17 and 18 of the cat visual cortex. Journal of Physiology (London), 311, 623-655.

Graham, C. H. (1965). Vision and visual perception. New York: Wiley.

HALPERN, D. L., \& BLAKE, R. (in press). Stereoacuity is a power function of contrast. Perception.

HeckmanN, T., Schor, C. M. (in press). Is edge information for stereoacuity spatially channeled? Vision Research.

Howard, I. P. (1982). Human visual orientation. New York: Wiley. Kaufman, L., \& ARDiti, A. (1976). The fusion illusion. Vision Research, 16, 535-543.

KerTesz, A. E. (1972). The effect of stimulus complexity on human cyclofusional response. Vision Research, 12, 699-704.

LEGGE, G., \& GU, Y. (in press). Stereopsis and contrast. Vision Research.

LE Grand, Y. (1967). Form and space vision (M. Millodot \& G. G. Heath, Trans.). Bloomington: Indiana University Press.

LeviTT, H. (1971). Transformed up/down methods in psychoacoustics. Journal of the Acoustical Society of America, 49, 467-477.

Mrtchell, D. E. (1966). A review of the concept of "Panum's fusional area." American Journal of Optometry, 43, 387-401.

ONo, H., \& BARBeIto, R. (1982). The cyclopean eye vs. the sightingdominant eye as the center of visual direction. Perception \& Psychophysics, 32, 201-210.

Panum, P. (1858). Physiologische Untersuchungen uber das Sehen mit zwei Augen. Kiel, West Germany: Schwers'sche Buchhandlung.

Pettigrew, J. D., Nikara, T., \& Bishop, P. O. (1968). Binocular interaction in single units in cat striate cortex: Simultaneous stimulation by single moving slits with receptive fields in correspondence. Experimental Brain Research, 6, 391-410.

ROSE, D., \& BLAKE, R. (1988). Distortions of perceived directions of diplopic images. Investigative Ophthalmology and Visual Science, 29(Suppl.), 411.

Rose, D., Blake, R., \& Halpern, D. L. (1988). Disparity range for binocular summation. Investigative Ophthalmology \& Visual Science, 29, 283-290.

SChor, C. M., \& HeckmanN, T. (in press). Interocular differences in contrast and spatial frequency: Effects on stereopsis and fusion. Vision Research.

SChor, C. M., HeckmanN, T., \& TYler, C. W. (in press). Binocular fusion limits are independent of contrast, luminance gradient and component phases. Vision Research.

SCHOR, C. M., \& TYLER, C. W. (1981). Spatio-temporal properties of Panum's fusional area. Vision Research, 21, 683-692.

Schor, C. M., Woon, I., \& OGaWa, J. (1984). Binocular sensory fusion is limited by spatial resolution. Vision Research, 24, 661-665.

SHERdY, J., FrY, G. (1979). The perceived direction of the binocular image. Vision Research, 19, 201-211.

TYLER, C. W. (1975). Spatial organization of binocular disparity sensitivity. Vision Research, 15, 583-590.

WeSTHeimer, G., \& McKeE, S. P. (1977). Spatial configurations for visual hyperacuity. Vision Research, 17, 941-947.

(Manuscript received June 6, 1988; revision accepted for publication October 3,1988 .) 